\title{
Distances to Galactic methanol masers
}

\author{
Kazi L. J. Rygl*i \\ Max-Planck-Institut für Radioastronomie, Germany \\ E-mail: kazi@mpifr-bonn.mpg.de
}

\section{Andreas Brunthaler}

Max-Planck-Institut für Radioastronomie, Germany

E-mail: brunthal@mpifr-bonn.mpg.de

\section{Karl M. Menten}

Max-Planck-Institut für Radioastronomie, Germany

E-mail: kmenten@mpifr-bonn.mpg.de

\section{Mark J. Reid}

Harvard-Smithsonian Center for Astrophysics, USA

E-mail: reidecfa.harvard.edu

\section{Huib Jan van Langevelde}

Joint Institute for VLBI in Europe, the Netherlands

Sterrewacht Leiden, Leiden University, the Netherlands

E-mail: langevelde@jive.nl

\begin{abstract}
We present the first EVN parallax measurements of $6.7 \mathrm{GHz}$ methanol masers in star forming regions of the Galaxy. The $6.7 \mathrm{GHz}$ methanol maser transition is a very valuable astrometric tool, for its large stability and confined velocity spread, which makes it ideal to measure proper motions and parallaxes. Eight well-studied massive star forming regions have been observed during five EVN sessions of 24 hours duration each and we present here preliminary results for five of them. We achieve accuracies of up to $51 \mu$ as, which still have the potential to be improved by more ideal observational circumstances.
\end{abstract}

The 9th European VLBI Network Symposium on The role of VLBI in the Golden Age for Radio Astronomy and EVN Users Meeting September 23-26, 2008 Bologna, Italy

\footnotetext{
* Speaker.

$\dagger$ Member of the International Max Planck Research School for Astronomy and Astrophysics at the Universities of Bonn and Cologne.
} 


\section{Introduction}

In astronomy, accurate distances are a very important quantity to measure. First, the determination of many physical properties of objects, such as the size-scale, the mass, the luminosity and the age depend on the distance. For example, the distance towards the Orion Nebula, determined by a trigonometric parallax of radio continuum stars [6], turned out to be $10 \%$ smaller than previously assumed. The implications of this are a 10\% drop in the masses, $20 \%$ fainter luminosities and 20-30\% younger ages for the stars in the field. Moreover, one also needs distances on a large scale to study the structure of our Galaxy.

Unfortunately, accurate distance measurements are very difficult. The only method which allows an unbiased measure of distance is trigonometric parallax, which is simply a geometrical method, and therefore free of any assumptions. To achieve the stability needed for optical wavelengths, parallax measurements require space-borne observations. Historically, the first dedicated optical satellite for this purpose was Hipparcos [7], which had an accuracy of 0.8-2 mas, allowing it to measure distances to $\sim 200 \mathrm{pc}$ (with error bars of 20\%). However, this successful experiment could reach only a tiny part of the Milky Way. A new optical astrometry satellite GAIA, to be launched in 2012, will be able to attain accuracies of $20 \mu$ as, and therefore provide important results on Galactic-scale distances. Despite the high accuracy reachable in the optical, GAIA will suffer from dust extinction, especially towards the large and dense dust lanes in the Galactic plane. It is in these regions that radio astronomy can provide an important complement to GAIA; radio wavelengths do not suffer from dust extinction and VLBI phase referencing techniques can provide accuracies up to $10 \mu$ as for obtaining accurate (with error bars of $10 \%$ ) distances to $10 \mathrm{kpc}$ (see for example [3],[12] and [9]). This is up to a 100-fold improvement on Hipparcos!

To measure the trigonometric parallax one requires very strong and compact sources. Masers are the ideal targets, found frequently in (dusty) star formation regions (SFRs) and AGB stars, objects whose distances one wants to know. Water masers at $22 \mathrm{GHz}$ inhabit both these regions, while methanol masers (12 and $6.7 \mathrm{GHz}$ ) are exclusively associated with high mass SFRs. Water and $6.7 \mathrm{GHz}$ methanol masers can be very strong, with peak fluxes of the order of a kilojansky, but because of their stability and confined velocity spread methanol masers are preferred for astrometry. Water masers are often associated with outflows in SFRs [2], which is manifested by a large velocity spread. Methanol masers are thought to be pumped by emission from warm dust, heated by a young stellar object and have velocity spreads of maximally $\sim 20 \mathrm{kms}^{-1}$, but frequently much smaller [11]. The frequency difference between water and methanol masers results in different sources of disturbance for each of the transitions. At higher frequencies the signal is more affected by tropospheric delay errors than at lower frequencies, where the ionosphere is typically more dominant. Also, the positional accuracy of the quasar one requires for phase referencing depends on frequency. At high frequencies one observes the more optically thick part of the quasar, while at lower frequencies one is dominated by the optically thin part and hence one can encounter more extended structure.

When the project began in 2006, the EVN was the only VLBI network that had receivers for observing the $6.7 \mathrm{GHz}$ maser transition. It offered a unique opportunity to start a project measuring the parallaxes and proper motions towards eight well known SFRs with strong methanol masers. Accurate distances towards these targets will have an important impact on the study of massive star 
formation in these regions.

\section{Observations and data reduction}

The observations were performed at five epochs using the EVN between 2006, June and 2008, March. The antennas participating in this experiment were Jodrell Bank, Westerbork in single dish mode, Effelsberg, Medicina, Torun, Onsala, Noto, Hartebeesthoek, Cambridge (first 3 epochs) and one VLA antenna (last epoch). Each observation lasted 24 hours and made use of geodetic-like observations to calibrate the tropospheric delays. A typical observing run contained 4 geodetic blocks of $\sim 1$ hour every 6 hours, 4 blocks of fringe finders of $\sim 10$ minutes, also every 6 hours, and 4 blocks of maser/quasar observations, spending on average between $\sim 0.9$ and $\sim 1.2$ hours on each maser. The difference in observing time per maser depends on the sky position; half of our targets are low declination sources, and have a limited visibility.

Using the technique of phase-referencing, each maser was observed in a cycle with one (or two) nearby $\left(\sim 1^{\circ}-2^{\circ}\right)$ quasars. The observations were performed with a data rate of $512 \mathrm{Mbps}$. The data were correlated at the Joint Institute for VLBI in Europe (JIVE), using an integration time of 0.5 seconds affording a field of view of 1.2 arc minutes (limited by time smearing). The maser data were correlated using one IF with high spectral resolution, with a bandwidth of $8 \mathrm{MHz}$ and a channel separation of $7.81 \mathrm{kHz}$, resulting in a spectral resolution of $0.35 \mathrm{kms}^{-1}$ at $6.7 \mathrm{GHz}$. The quasar sources were correlated in continuum mode with $8 \mathrm{IFs}$, of $8 \mathrm{MHz}$ bandwidth with a channel separation of $0.25 \mathrm{MHz}$.

The data reduction was done using the Astronomical Image Processing System (AIPS). The geodetic-like observations were reduced separately, and tropospheric delays were estimated for each antenna (see [8]) and corrected with the task 'CLCOR'. The data were reduced following the EVN guidelines, applying parallactic angle and ionospheric delay corrections. Amplitudes were calibrated using system temperature measurements and standard gain curves. The correction for the Earth rotation was done with the task 'CVEL'. Since we observed strong masers we could use them as the phase reference, and the solutions were transferred in a created 8IF dataset to the continuum data. The quasar and maser positions were extracted by fitting $2 \mathrm{D}$ gaussians to the maps. The differences in position between a maser and quasar pair were fitted, yielding the parallax and proper motions. When more than one maser spot was used for the fit, the error was multiplied by $\sqrt{N}$, where $N$ is the number of spots. This allows the random errors to be $100 \%$ correlated, and is therefore a conservative and safe approach.

\section{Preliminary results}

\subsection{L1287}

L1287 is an interesting SFR, where CO observations [13] clearly indicate a bipolar outflow. An $\mathrm{NH}_{3}(1,1)$ study [1] shows that there is cold and dense gas at the base of the outflow. In 2006, Imai et al. found evidence for a disk from the distribution of the radial velocities of water masers [4]. We measure a parallax of $1.12 \pm 0.06$ mas (shown in Figure 1), corresponding to a distance of $891 \pm 48 \mathrm{pc}$ - very close to the kinematic distance of $850 \mathrm{pc}$ obtained from the ammonia spectral lines. 

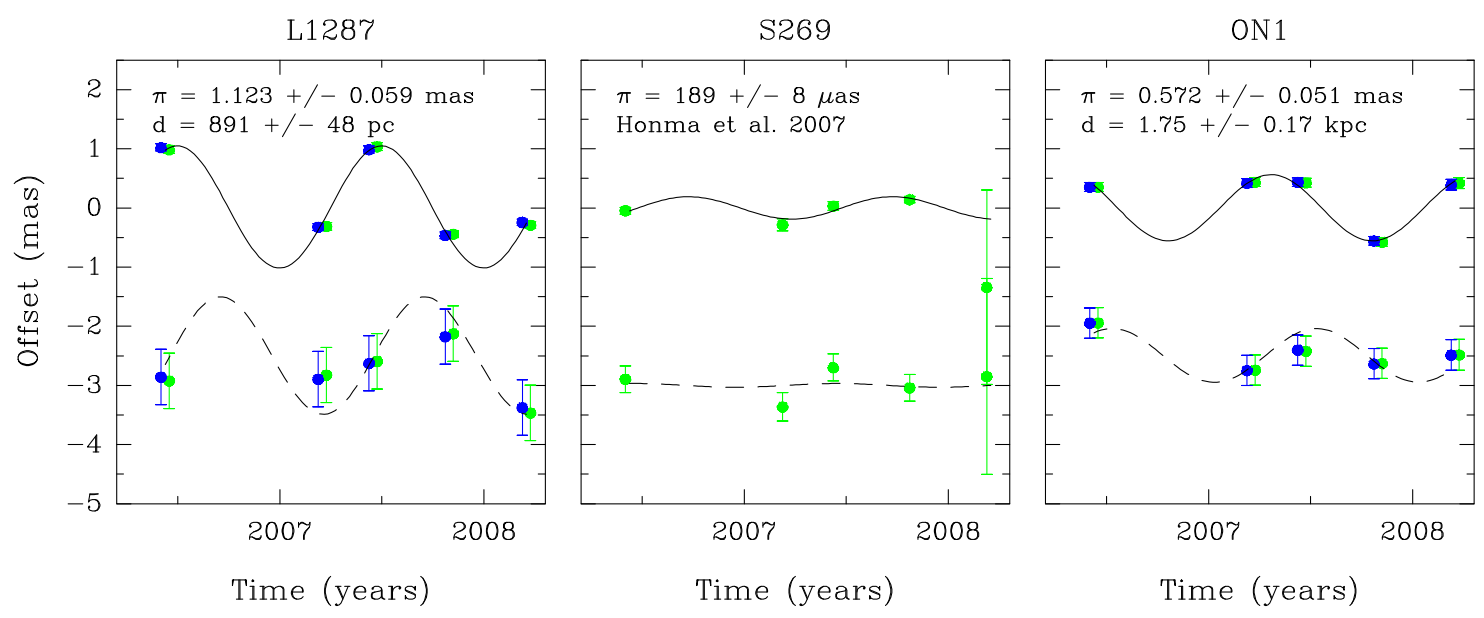

Figure 1: The preliminary results for L1287, S269 and ON1. The Right Ascension (solid line) and declination (dashed line) signature of the parallax are shown. Each maser channel which was used for the parallax fitting is indicated by a different color.

\subsection{9}

In 2007, Honma et al. measured the trigonometric parallax of water masers to this SFR in the outer Galaxy to be $189 \pm 8 \mu$ as corresponding to a distance of $5.28 \pm 0.24 \mathrm{kpc}$ [3]. We were not able to reach the necessary accuracy to verify the results of Honma et al. with our observations (Figure 1 shows the parallax of Honma et al. and our measurements). Nevertheless, we can study the methanol proper motions and compare them to the proper motions obtained from the water maser to test intrinsic differences between the methanol and water maser distribution in this SFR.

\subsection{Overview}

A summary of the preliminary results is given in Table 1 . Here, we briefly discuss the remaining results. We measure the parallax of ON1 with an accuracy of $51 \mu$ as, corresponding to a distance of $1.75 \pm 0.17 \mathrm{kpc}$ (shown in Figure 1). This is the highest accuracy we obtained due to a favorable sky position and a good sampling of the parallax signature (peak, valley). For NGC281W we obtained the largest distance of $2.53 \pm 0.78 \mathrm{kpc}$, which is in excellent agreement with the water maser results [10]. For S252 we did not manage to detect the quasar in some epochs. However, S252 had a recent $12 \mathrm{GHz}$ methanol maser parallax determined [9]. The last three sources (not in the Table), S255, L1206 and MonR2, are being worked on and we do not discuss them here.

\section{Discussion}

We have demonstrated that one can measure the trigonometric parallax of $6.7 \mathrm{GHz}$ methanol masers using the EVN. Good results were obtained for sources of higher declinations $\left(\delta>25^{\circ}\right)$. The accuracy reached in this experiment is $\sim 50-100 \mu$ as, which is $10-20$ times better than Hipparcos. The accuracy of parallax measurements of the $6.7 \mathrm{GHz}$ maser transition with the EVN can be improved by taking notice of the following. Our project was large; it consisted of eight masers, all at different R.A.s and declinations. In the five sessions, we did not manage to have the ideal parallax signature coverage for each maser (compare L1287 with ON1 in Figure 1), for which 
Table 1: Preliminary Distances AND Literature VAlues

\begin{tabular}{lllll}
\hline \hline SFR & $\begin{array}{l}\text { Parallax } \pm \text { error } \\
\text { mas }\end{array}$ & $\begin{array}{l}\mathrm{D}_{\text {parallax }} \pm \text { error } \\
\mathrm{kpc}\end{array}$ & $\begin{array}{l}\mathrm{D}_{\text {kinetic }} \\
\mathrm{kpc}\end{array}$ & $\begin{array}{l}\text { Literature D } \\
\mathrm{kpc}\end{array}$ \\
\hline L1287 & $1.123 \pm 0.059$ & $0.891 \pm 0.049$ & 0.850 & $\mathrm{D}_{\text {kin }} ;[1]$ \\
ON1 & $0.572 \pm 0.051$ & $1.75 \pm 0.17$ & 1.8 & $\mathrm{D}_{\text {kin }} ;[5]$ \\
NGC281-W & $0.395 \pm 0.1$ & $2.53 \pm 0.78$ & 2.7 & $2.8 ;[10]$, parallax \\
S269 & - & - & 3.0 & $5.3 ;[3]$, parallax \\
S252 & - & - & 4.4 & $2.1 ;[9]$, parallax \\
\hline \hline
\end{tabular}

one prefers to sample the peaks. Often, as for L1287, some sessions were near the zero-signature of the parallax, decreasing the quality of the fit. Another problem encountered in the project was the large number (50\%) of low-declination sources. We did not manage to observe them as long as their high declination counterparts. This, combined with their low elevations, resulted in a poor $u, v$ coverage.

As in previous parallax studies, the position uncertainties in declination are significantly worse than in Right Ascension (see for example L1287 in Figure 1). First, the errors introduced by the atmosphere are larger in declination. Second, the shape of the beam is more elongated in declination than in Right Ascension. For example, the source S269 has a beam of $7.7 \times 3.7$ mas with a position angle of $55^{\circ}$.

Finally, we would like to touch upon the geodetic-like observations. We find an improvement in the calibration for most of the cases. However, in a few cases the corrections did not improve the calibration. We suspect that here the ionospheric delay plays a more important role, and actually we degrade the calibration by wrongly interpreting the determined delay as of tropospheric origin. In these cases, we did not apply the geodetic-like correction. A dual frequency observation mode, as employed in geodetic observations to calibrate the ionosphere, is not possible with most of the EVN antennas. Also switching receivers is impossible. Therefore, determining the $\mathrm{S} / \mathrm{N}$ with or without a geodetic-like correction in each observation is the best option for now.

In conclusion, we have shown that 6.7 methanol masers, observed with the EVN, are a well suited tool for astrometry. The fact that the VERA array was recently equipped with $5 \mathrm{GHz}$ receivers is a sign for a growing enthusiasm for $6.7 \mathrm{GHz}$ methanol masers. The final astrometry of our sources will be used to reevaluate the SFRs' astrophysics in the light of the new distance determinations, and to study the 3D velocities of the SFRs relative to Galactic motion.

\section{References}

[1] Estalella, R., Mauersberger, R., Torrelles J. M. et al., 1993, ApJ 419, 698

[2] Hachisuka, K., Brunthaler, A., Menten, K. M. et al., 2006, ApJ 645, 337

[3] Honma, M., Bushimata, T., Choi, Y. K. et al., 2007, PASJ 59, 889

[4] Imai, H., van Langevelde, H. J. \& Umemoto, T., 2006 in: Proceedings of 8th EVN Symposium

[5] MacLeod, G. C, Scalise, E. Jr., Saedt, S. et al., 1998, AJ 116, 1897 
[6] Menten, K. M., Reid., M. J., Forbrich, J. \& Brunthaler, A., 2007, A\&A 474, 515

[7] Perryman, M. A. C., Lindegren, L., Kovalevsky, J. et al., 1995, A\&A 304, 69

[8] Reid, M. J. \& Brunthaler, A., 2004, ApJ 616, 872

[9] Reid, M. J., Menten, K. M., Brunthaler, A. et al., 2008, ApJ in press, arXiv:0811.0595

[10] Sato, M., Hirota, T., Honma, M. et al., 2008, PASJ 60, 975

[11] Sobolev, A. M., Cragg, D. M., Ellingsen, S. P et al., 2007 in: Proceedings IAU Symposium 242, 81

[12] Xu, Y., Reid, M. J., Zheng, X. W. \& Menten, K. M. 2006, Science 311, 54

[13] Yang, J., Umemoto, T., Iwata, T. et al., 1991, ApJ 373, 137 